\title{
Melting Behaviour for Gels Formed from the Solution of Chloroform and ABA Triblock Copolymer comprised of Poly (L-leucine) and Poly(ethylene glycol)
}

\author{
Yutaka Tanaka and Yoichi Takayanagi \\ Department of Materials Science and Engineering, Faculty of Engineering, University of Fukui, Fukui 910-8507, Japan \\ Fax: 81-(0)776-27-8767, e-mail: tanaka@matse.u-fukui.ac.jp
}

\begin{abstract}
ABA triblock copolymer comprised of poly (L-leucine) as the A segment and polyethylene glycol as the B segment was prepared by ring opening polymerisation of $N$-carboxyanhydride of L-leucine using the amine-terminated poly(ethylene glycol) as an initiator. Melting temperature for the gels prepared through the solution of the copolymer and chloroform was observed by inverting the test tube. The results of observation were interpreted in terms of Eldridge - Ferry plot.

Key words: triblock copolymer, poly(L-leucine), PEG, gel, Eldridge-Ferry plot
\end{abstract}

\section{INTRODUCTION}

Physical gels usually have complex junction zones. To describe properties of such complex junctions, many research works adopts the notion of junction multiplicity defined by the number of chains combined to a single junction. $[1,2] \quad$ One of the simple procedure to analyse the junction zone is the Eldridge - Ferry plot, which uses the relation between the logarithm of gel concentration $(c)$ and the melting temperature $\left(T_{\mathrm{m}}\right)$, to give the total enthalpy of junction fusion by finding the slope of $\log c$ against $1 / T_{\mathrm{m}}$

Although the method of Eldridge - Ferry plot is used for many works of the melting of gel(see ref. 3-7), so far the application to block copolymer gel is limited. For block copolymers, including telechelic hydrophobically modified hydrophilic polymer, physical properties are currently researched with wide range attentions; those are rich morphological textures in solid, macromolecular assembly to generate polymeric micelles and ordered structure in selective solvents, potential applications as compartibilisers in polymer blends and as biomedical materials.[8-16] In particular, ABA triblock copolymer is the one of the key technologies to realise self-assembly both in solid and in solution, because it has well-defined structures on the nanometer scale and the capacity for the control of self-organisation in such systems by the combination of opposite interactions that can result in processing order over multiple length scales.

In this study, the melting of the physical gel formed by $\mathrm{ABA}$ triblock copolymer and the organic solvent was examined in terms of the relation between the size of junction zone and total enthalpy of junction fusion from the viewpoint of Eldridge - Ferry plot. The system consists of the synthetic water-soluble semicrystalline polymer, poly(ethylene glycol) as the B segment, that is, one of the well-studied homopolymer. The second macromolecule is of biological origin and is based on the well-known polypeptide poly(L-leucine) as the A segment, where intermolecular hydrogen bonds stabilise the secondary structures.

The triblock copolymer comprised of poly(ethylene glycol) and poly(L-leucine) is widely researched at present from several practical points of view. Those are, for example, materials concentration technology, materials removing, encapsulation in medical engineering, biodegradable carrier, protein modification, wound dressing.[15, 17-21] A key feature of this copolymer is in designing the controllable network structure. The factors are, the balance in lengths between the midblock and the side blocks, the whole length of polymer chain, kinds of $\alpha$-amino acid in the side blocks, chain stiffness / flexibility for both blocks, the balance of hydrophilicity / hydrophobicity. These properties will affect such properties that gel formation, the solubility to organic solvents, and therefore the efficiency for materials concentration, biodegradability, gas permeability. Gel melting temperature and the enthalpy of junction fusion investigated in this study will be expected to support the designing of controllable network for the respective practical purpose. With these backgrounds, $T_{\mathrm{m}}$ of ABA triblock copolymer composed of poly(L-leucine) as A segment and poly(ethylene glycol) as B segment is investigated in this study; abbreviations of PLL, PEG and PLLPEG are used to refer to the segments and the triblock copolymer respectively.

\section{EXPERIMENTAL}

Amine-terminated polyethylene glycol $\left(M_{\mathrm{n}}=3350\right.$, Sigma) was purchased and used without further purification. Bis(trichloromethyl) carbonate, Lleucine, isobutylamine used are commercially available. $N, N^{\prime}$ - Dimethylformamide(DMF) was dehydrated by $\mathrm{CaH}_{2}$ and subsequently stored over molecular sieves (4A). Tetrahydrofuran(THF), benzene and hexane were dehydrated by sodium wire and stored in sealed glass tubes.

In the synthesis of L-leucine $N$-carboxyanhydride (leucine-NCA), L-leucine was suspended in THF. Bis(trichloromethyl) carbonate of three time excess amount to L-leucine in molar ratio was dissolved in THF and added to the suspension. The reaction mixture was heated to $50^{\circ} \mathrm{C}$ under stirring. After the reaction mixture became transparent(approximately $30 \mathrm{~min}$ ), THF was evaporated under reduced pressure. The obtained leucine-NCA was recrystallised three times from a mixture of THF and hexane. Then, powdered 
leucine-NCA was obtained by lyophilisation through benzene solution.

The copolymers were synthesized by the following procedure. The ring-opening polymerisation of leucine-NCA was carried out for $24 \mathrm{~h}$ at $50^{\circ} \mathrm{C}$ under a nitrogen atmosphere, initiating from an amine-terminated polyethylene glycol( $5 \% \mathrm{w} / \mathrm{v})$ in DMF, while varying the leucine-NCA composition in the feed. The products were washed with hexane then PLL-PEG-PLL triblock copolymers were obtained lyophilisation through benzene solution. The yield was around $60 \%$ on the basis of PEG recovery. The chemical structures were confirmed by infrared spectoscopy (IR) and proton nuclear magnetic resonance $\left({ }^{1} \mathrm{H}\right.$ NMR $)$. Poly(L-leucine) was prepared by the same method as used for the copolymer with isobutylamine as an initiator.

The ${ }^{1} \mathrm{H}$ NMR spectra for leucine-NCA, copolymer and poly(L-leucine) were measured at room temperature in a solvent of DMSO- $d_{6}$ on a JEOL lambda500 FT NMR spectrometer to estimate the copolymer compositions and the molecular weights of the PLL segment.

The IR spectra were measured on a Nicolet Inc. Magna spectrometer between 4000 and $400 \mathrm{~cm}^{-1}$. $\mathrm{X}$-ray diffractions (XRD) were recorded on an X-ray diffractometer (RINT-2100) equipped with a fibre specimen attachment.

\section{RESULTS AND DISCUSSION}

Synthesis and Characterisation of Triblock copolymers.

To prepare PLL-PEG-PLL triblock copolymers with varying degrees of polymerisation $(D P)$ of the PLL segments, we carried out the ring-opening polymerisation of leucine-NCA initiating from the amine-terminated PEG. The molar ratios of leucine-NCA and the terminal amine group in each chain end in the feed were 5,10 and 50 in order to control DP of the PLL segment. The polymerisation proceeded homogeneously. The IR spectra of the copolymer showed the following vibration peaks: $3300 \mathrm{~cm}^{-1}\left(\mathrm{~m}, \mathrm{~N}-\mathrm{H}\right.$ amide), $2873 \mathrm{~cm}^{-1}(\mathrm{~s}, \mathrm{C}-\mathrm{H}), 1653 \mathrm{~cm}^{-1}$ (s, amide I), $1544 \mathrm{~cm}^{-1}$ (s, amide II), and 1467, 1353, $1255,1108,847,618 \mathrm{~cm}^{-1}$, while that of leucine-NCA showed vibration peaks of $1817,1753,944 \mathrm{~cm}^{-1}$ (s, ring structure) in addition to the peaks of the copolymer. That is, the reaction of leucine-NCA was completed as ascertained by tracing the characteristic carbonyl IR bands. The ${ }^{1} \mathrm{H}$ NMR spectra showed that all of the polymers presented well-defined NMR spectra

with similar characteristics; $\delta=8.3,6.6 \mathrm{ppm}(\mathrm{H}, \mathrm{m},-\mathrm{NH}-)$, 4.3, 3.3ppm $\left(3 \mathrm{H}, \mathrm{m},-\alpha-\mathrm{CH}_{2}-\right.$ and $\left.-\mathrm{CH}-\right), 3.6 \mathrm{ppm}(4 \mathrm{H}$, $\left.\mathrm{s},-\mathrm{CH}_{2} \mathrm{CH}_{2} \mathrm{O}-\right), 1.8 \mathrm{ppm}(6 \mathrm{H}$, broad, isopropyl, end-NH and $\left.-\beta-\mathrm{CH}_{2}-\right), 0.9 \mathrm{ppm}\left(3 \mathrm{H}, \mathrm{s},-\mathrm{CH}_{3}\right)$, where the strength of the peak at $3.6 \mathrm{ppm}$ is roughly constant among different samples. These results confirmed that copolymerisation by the ring-opening reaction with
leucine-NCA resulted in monodisperse triblock copolymers containing two PLL blocks attached at both PEO chain terminals.

The copolymer composition was determined using the intensities of peaks in ${ }^{1} \mathrm{H}$ NMR spectrum with respect to the repeating number of PEG segment which is assumed to be equivalent to that of amine-terminated polyethylene glycol used as an initiator of the copolymerisation. Table I shows comparisons between the intensity measured directly from the NMR spectrum and the value expected from the chemical formulae for samples copolymerised with the molar ratio of leucine-NCA of 5, 10 and 50 in the feed for the reactions at both ends; they are denoted by PLLPEGx, $\mathrm{x}=5,10$ and 50 respectively. Values of $h_{\text {cal }}$ were obtained with assuming $D P$ of the each end is equivalent with the feed molar ratio. In the examination of Table I, $h_{\text {integ }}$ and $h_{\text {cal }}$ showed large differences for ${ }^{1} \mathrm{H}$ of $-\mathrm{CH}_{2} \mathrm{CH}_{2} \mathrm{O}$-, i,e. ${ }^{1} \mathrm{H}$ contained in PEG backbone. However, little attention was paid on this result. Due to the repeating number of PEG backbone, ${ }^{1} \mathrm{H}$ of $-\mathrm{CH}_{2} \mathrm{CH}_{2} \mathrm{O}$ - are in large number. Therefore it seems to be difficult to lay integral curve clearly to enclose the NMR peak. As a result of the ${ }^{1} \mathrm{H}$ NMR intensity, it can be said that leucine-NCA reacted stoichiometrically in the copolymerisation, although $h_{\text {integ }}$ is still inconsistent with $h_{\text {cal }}$ in some ${ }^{1} \mathrm{H}$.

In order to lay more confidence on $D P$ of PLL segment, PLL homopolymer was prepared with the molar ratio of leucine-NCA of 10 against the initiator(isobutylamine) in the feed using the same procedure as in the copolymerisation. Fig. 1 shows results of the viscosity measurement for PLL homopolymer carried out with trifluoroacetic acid as the solvent at $25^{\circ} \mathrm{C}$. Consequently, an intersection point was found among Huggins plot, Fuoss-Mead plot and Bill-Mayer plot to give the intrinsic viscosity; $[\eta]=10.83$. The result was examined with the relationship of viscosity and the number average 
molar mass shown below obtained for poly(L-leucine) in trifluoroacetic acid.[22]

$$
[\eta]=1.68 \times 10^{-2} \times M_{\mathrm{n}}^{0.93} \quad \text { (1) }
$$

$D P$ value obtained for this sample was 8.6 which is approximately close to the molar ratio of the charged monomer to the initiator.

In order to obtain any information to imply ordered structures of the copolymer, the powdered samples were investigated by XRD study. PLL homopolymer and amine-terminated polyethylene glycol were also investigated for comparison (see Fig.2). The diffraction peaks are summarised in Table II with $d$-spacing.

A comparison of the XRD showed the following information. There are some ordered structures in PLL with the peaks at 12.0 and

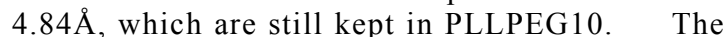
peak intensity at $4.84 \AA$ relative to that at $12.0 \AA$ for PLLPEG10 is lower than the intensity of PLL; showing that the density of the ordered structures decreased by the copolymerisation. PEG segment is crystallised in the copolymer.

With respect to the ordered structure in PLL, both the $\alpha$-herix and $\beta$-structure have been reported; with the increase in $D P$ the structure will change from $\beta$-sheet to $\alpha$-herical conformation.[22] The reported values of the unit cells for PLL are; $a=4.80, b=23.56, c=6.88 \AA$ of the orthorhombic unit cell for the antiparallel $\beta$-structure, and $a=13.22 \AA$ of the hexagonal unit cell for the $\alpha$-herix. Taking account that the peak at $12.0 \AA$ for PLL shown in Fig. 2 can come from the reflection at the (020) plane, and therefore $d$-spacing of $12.0 \AA$ corresponds with the half of $b$, the powdered sample used in XRD is considered to form $\beta$-structure.

\section{Gel Formation and Melting Temperature}

Gel formation was examined concerning PLLPEG10 for various organic solvents to be found that solutions of chloroform and 1,4-dioxane in the moderate concentration form transparent gels. The gel of PLLPEG10dioxane showed a melting temperature around $100^{\circ} \mathrm{C}$. In addition, the gels prepared by mixed solvents of dioxane and methanol varied the melting temperature from room temperature to around $100^{\circ} \mathrm{C}$ according to the mixing ratio. These gelation behaviours must be examined in terms of the solubility of both A and B segments,

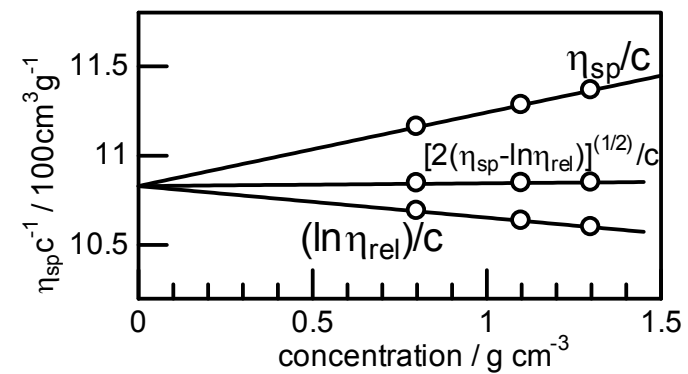

Fig.1. Results of viscosity measurements for the PLL homopolymer solution of trifluoroacetic acid at $25^{\circ} \mathrm{C}$. $\left[2\left(\eta_{\mathrm{sp}}-\ln \eta_{\mathrm{rel}}\right)\right]^{(1 / 2)} / c$ : Billmayer plot, $\eta_{\mathrm{sp}} / c$ :Huggins plot, $\left(\ln \eta_{\mathrm{rel}}\right) / c$ :Fuoss-Mead plot.

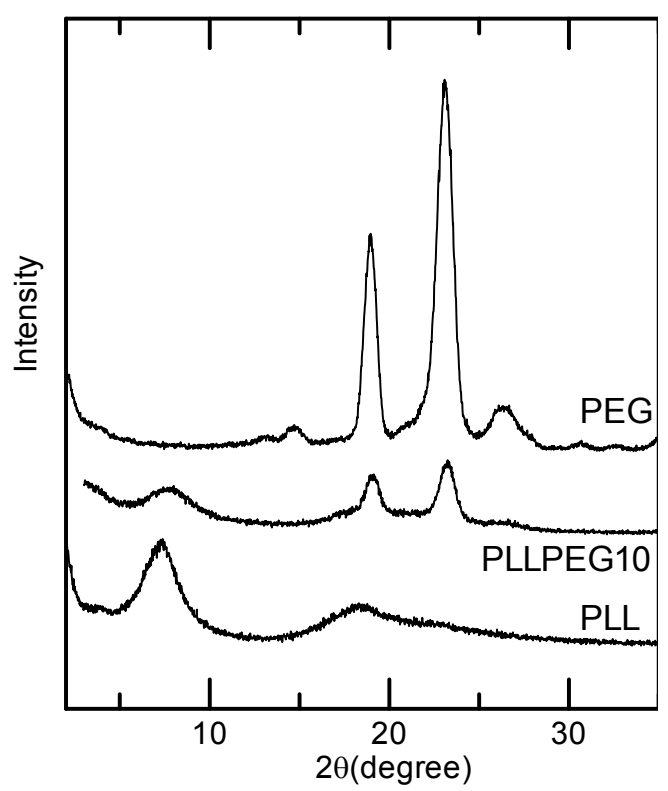

Fig.2 Wide-angle X-ray diffraction patterns of powdered samples of amine-terminated PEG, PLLPEG10 and PLL homopolymer.

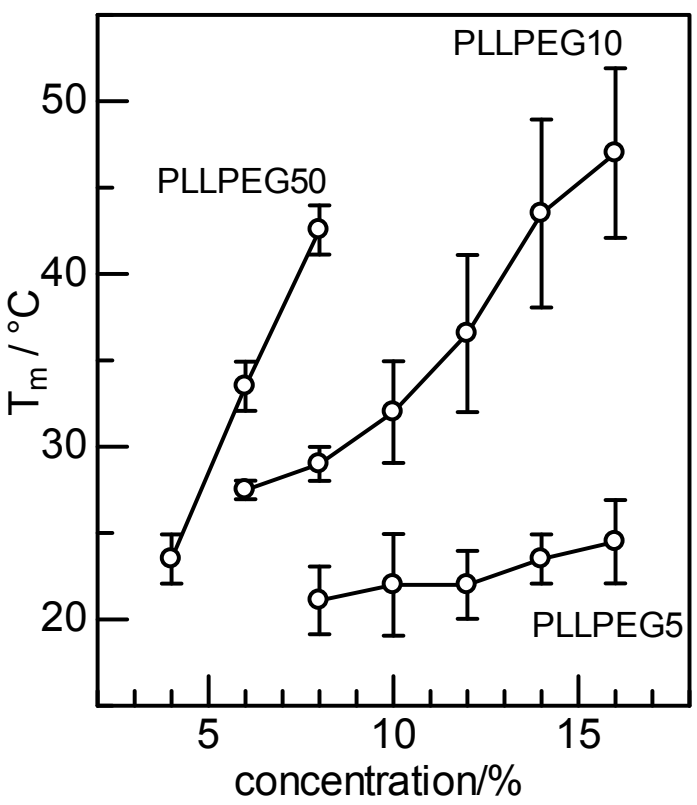

Fig. 3. The relation of the melting temperature and the concentration for triblock copolymer gels of chloroform solutions.

which is important research scope. It is necessary that further investigation will be carried on to clarify why the solution forms a gel on a particular solvent.

The melting was observed with the method to invert a test tube for the gels of PLLPEGxchloroform, $\mathrm{x}=5,10,50$. In the measurements the solution was (1) poured into a sealed test tube; (2) heated to around $70^{\circ} \mathrm{C}$ to melt the gel; (3) cooled with ice water and stored in a refrigerator; and heated again using water bath to be observed. The melting behaviour was complicated; the partial melting took place. 
Therefore the observation was repeated and the averaged value was plotted against the solution concentration (weight \%) with an error bar in Fig.3. In particular, the gels which concentrations are higher than $10 \%$ showed partial melting significantly.

Although there are error bars, $T_{\mathrm{m}}$ increased with the increase in $D P$ of PLL segment as compared among gels of same concentration. The lowest concentrations of gels used in this measurement were $8 \%, 6 \%$ and $4 \%$ for PLLPEGx, $\mathrm{x}=5,10,50$ respectively. The solution whose concentration was lower than this did not form gel; that is, the minimum gelation concentration decreased with $D P$ of PLL segment. It can be considered that the minimum gelation concentration is the reason that the relations of $T_{\mathrm{m}}$-concentration in Fig. 3 don't intersect together among three PLLPEG.

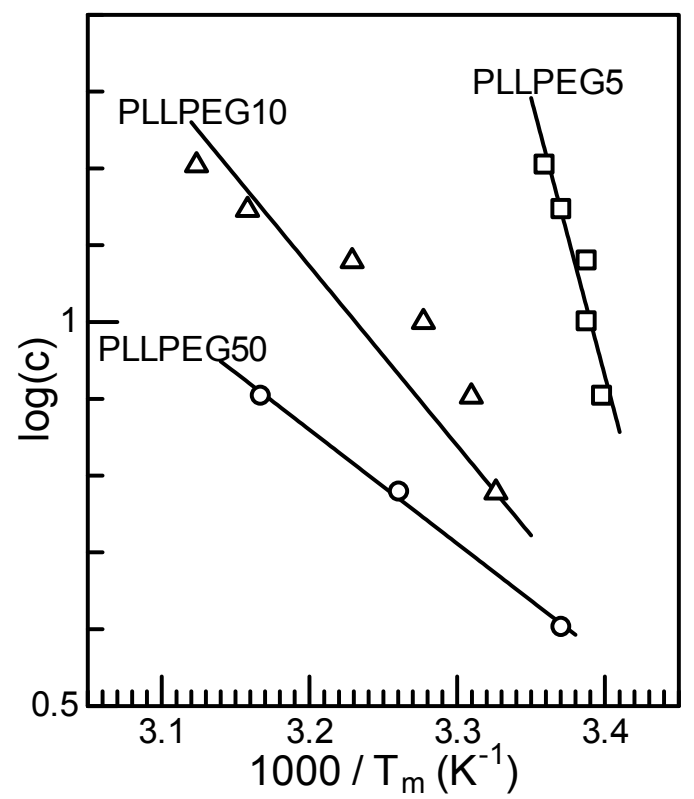

Fig. 4. Eldridge-Ferry plots for triblock copolymer gels of chloroform solutions constructed through $T_{\mathrm{m}}$ data shown in Fig.3.

The results that $T_{\mathrm{m}}$ depended on $D P$ of PLL segment implies that PLL segments take part in the formation of junction zone. PLL segments are supposed to be able to aggregate together in solution by adding the information of ordered structure evidenced through XRD study.

Fig. 4 shows the Eldridge-Ferry plots for $T_{\mathrm{m}}$ shown in Fig.3. The enthalpy of junction fusion was evaluated as $139,45,28 \mathrm{~kJ} / \mathrm{mol}$ for PLLPEGx $x=5,10,50$ respectively, showing that the enthalpy decreased with the increase in $D P$. As a plausible interpretation for this result, the number of PLL chains combined to a junction can be considered; that is that number decreases with the order of PLLPEGx, $x=50,10,5$. The enthalpy of fusion becomes less as the junction includes less segments. However, the interpretation mentioned here is not proved without clarifying the structure of the junction zone of the gel formed by the solution of PLLPEG. It is necessary to be cautious before coming to the conclusion. Other investigation to measure the aggregation of PLL chains is indispensable to obtain the information for the relation of $D P$ and the heat of fusion of junction zone.

\section{REFERENCES}

[1] P. J. Skrzeszewska, F. A. de Wolf, M. W. T. Werten, A. P. H. A. Moers, M. A. Cohen Stuart, J. van der Gucht, Soft Matter, 5(10), 2057-2062 (2009).

[2] M. Takahashi, M. Shimazaki, J. Polym. Sci., Part B: Polym. Phy. 39(9), 943-946(2001).

[3] Hisao Kai Jen, Wei-Liang Chen, Yu-Ming Chang, Chwen-Tay Ke, J. Mat. Sci., 32(13), 3607-3611(1997).

[4] Huei-Hsiung Wang, Tien-Wei Shyr, Mei-Shan Hu, J. Appl. Polym. Sci. 73(11), 2219-2226 (1999).

[5] V. I. Lozinsky, A. L. Zubov, I. N. Savina, F. M. A. N. Plieva, J. Appl. Polym. Sci. 77(8), 1822-1831(2000).

[6] P. M. Gilsenan, S. B. Ross-Murphy, J. Rheol., 44(4), 871-883(2000).

[7] Ching-Feng Mao, J. Appl. Polym. Sci., 102(1), 663-673(2006).

[8] S. Tanaka, A. Ogura, T. Kaneko, Y. Murata, M. Akashi, Macromolecules, 37, 1370-1377 (2004).

[9] G. Floudas, P. Papadopoulos, H.-A. Klok, G. W. M. Vandermeulen, J. Rodriguez-Hernandez, Macromolecules, 36, 3673-3683(2003).

[10] T. Fujimoto, E. Yoshimoto, M. Annaka, Prog. Coll. Polym. Sci. 136, 77-85(2009).

[11] A. Noro, Y. Matsushita, T. P. Lodge, Macromolecules, 42, 5802-5810(2009).

[12] K. A. Aamer, H. Sardinha, S. R. Bhatia, G. N. Tew, Biomaterials, 25, 1087-1093(2004).

[13] T. A. Walker, J. J. Semler, D. N. Leonard, G. J. van Maanen, R. R. Bukovnik, R. J. Spontak, Langmuir, 18(22), 8266-8270(2002).

[14] Iwhan Cho, Jin-Baek Kim, Hyun-Jin Jung, Polymer, 44, 5497-5500(2003).

[15] H. Wang, X. Chen, Cai-Yuan Pan, Eur.

Polym. J., 43, 1905-1915(2007).

[16] W. Zhu, W. Xie, X. Tong, Z. Shen, Eur. Polym. J., 43, 3522-3530(2007).

[17] S. Tanimoto, N. Yagi, H. Yamaoka, Res. Lett. Mat. Sci., 597308(2009)

[18] Y. Ohya, H. Yamamoto, K. Nagahama, T. Ouchi, J. Polym. Sci., Part A: Polym. Chem., 47(15), 3892-3903(2009).

[19] H. Yokoi, S. Mizutani, T. Kinoshita, Trans. Mat. Res. Soc. Jpn., 27(3), 551-554(2002).

[20] J. K. Sakata, W. Shen, D. A. Tirrell, Polym. Mat. Sci. Eng. , 84, 812-813(2001).

[21] H. J. Kim, E. Y. Choi , J. S. Oh , H. C. Lee, S. S. Park, C. S. Cho, Biomaterials, 21(2), 131-141(2000).

[22] T. Komoto, K. Y. Kim, M. Oya, T. Kawai, Makromolekulare Chemie, 175, 283-299(1974).

(Received January 12, 2010; Accepted March 27, 2010) 